Nonsi ngul ar const rai nt s i n t i me- dependent vari at i onal pri nci ple for par amet ri zed wave f unct i ons

\begin{tabular}{|l|l|}
\hline 著者 & OHTA Kat suhi sa \\
\hline $\begin{array}{l}\text { j our nal or } \\
\text { publ i cat i on t it l e }\end{array}$ & I nt er nat i onal J our nal of Quant um Chemi stry \\
\hline vol une & 113 \\
\hline number & 2 \\
\hline page range & $161-170$ \\
\hline year & $2013-01-15$ \\
\hline URL & ht t p: //hdl . handl e. net /10258/00009465 \\
\hline
\end{tabular}




\title{
Nonsingular constraints in time-dependent variational principle for parametrized wave functions
}

\author{
Katsuhisa Ohta ${ }^{1, *}$ \\ ${ }^{1}$ Department of Applied Chemistry, Muroran Institute of Technology, \\ Muroran, Hokkaido 050-8585, Japan
}

\begin{abstract}
In this work we consider two conditions required for the nonsingularity of constraints in the timedependent variational principle (TDVP) for parametrized wave functions. One is the regularity condition which assures the static nonsingularity of the constraint surface. The other condition is the second-class condition of constraints which assures the dynamic nonsingularity of the constraint surface with a symplectic metric. Especially for analytic wave functions for complex TDVPparameters, the regularity and the second-class conditions become equivalent. The second-class condition for expectation values is reduced to the non-commutability of the corresponding quantum operators. The symplectic singularity of the equation of motion of TDVP (TDVP-EOM) is also shown to be a local breakdown of the second-class condition in an extended canonical phase-space.

PACS numbers:
\end{abstract}

*Electronic address: ohta@mmm.muroran-it.ac.jp 


\section{INTRODUCTION}

In quantum dynamics, the variational approach is one of the most practical methods to obtain time developments of wave functions with appropriate variational parameters[1]. Depending on forms of the functional, there are three types in the variational calculation, namely referred to Dirac-Frenkel's [2, 3], McLachlan's[4], and the time-dependent variational principle (TDVP)[5-10]. Especially the TDVP, based on the least-action principle, has attracted much interest since its theoretical framework is similar to that of classical mechanics. By utilizing the pseudo-classical structure, we have introduced constraints in the TDVP[11-13] according to the Dirac's constrained classical-mechanics[14, 15]. Constraints in variational approaches can be used for various purposes, such as to keep some formal symmetries of the system, to construct some physical situations, to analyze physical or chemical effects of some freedoms, and so on [16-19]. It is necessary, however, to require some conditions for nonsingular constraints as in constrained classical-mechanics[20-22].

In this work, we consider two types of such conditions in the TDVP to construct a nonsingular constraint surface. One is the regularity condition of constraints. This condition, as discussed in $\S \mathrm{II}$, constructs the nonsingular static structure of the constraint surface. The other condition is the second-class condition of constraints which assures the nonsingular dynamic structure of the constraint surface with a so-called symplectic metric[7] as discussed in §III. Especially for analytic wave functions for the complex TDVP-parameters[8, 10], we show some simple features of the TDVP. The regularity condition becomes equal to the second-class condition. Moreover, the Poisson bracket of expectation values is proven to be equal to the expectation of the commutator of corresponding quantum operators[12] as shown in $\S I V$. Some examples are given for second-class conditions in $\S \mathrm{V}$. As one of the examples, the symplectic singularity in the equation of motion of TDVP (TDVP-EOM) [23, 24] is shown to be a local breakdown of the second-class condition with a numerical example. The section VI gives the summary of the present work. 


\section{REGULARITY CONDITION FOR STATIC NONSINGULARITY OF CON- STRAINTS}

In this section we consider the regularity condition of constraints. The regularity condition assures the independence of freedoms to be frozen in variational calculations and constructs the nonsingular constraint surface.

We parametrize wave functions $\Psi$ through real-valued parameters $\left\{\alpha_{i}\right\}_{i=1, N}$. The variational freedom $\delta \Psi$ is expanded and restricted within a predetermined region of Hilbert space by the parameters $\left\{\alpha_{i}\right\}_{i=1, N}$ as

$$
|\delta \Psi\rangle=\sum_{i=1}^{N}\left|\frac{\partial \Psi}{\partial \alpha_{i}}\right\rangle \delta \alpha_{i}
$$

We here assume that the local bases $\left\{\left|\frac{\partial \Psi}{\partial \alpha_{i}}\right\rangle\right\}_{i=1, N}$ are independent as

$$
\operatorname{Rank}[\boldsymbol{S}]=N \text {, }
$$

by the Gramian matrix $(\boldsymbol{S})_{i j}=\left\langle\frac{\partial \Psi}{\partial \alpha_{i}} \mid \frac{\partial \Psi}{\partial \alpha_{j}}\right\rangle$.

In quantum mechanics, constraints should be applied to the wave function. We here assume that such a constraint can be also specified by another real parameters $\left\{f_{a}\right\}_{a=1, M}$ as

$$
\left|\delta \Psi_{f}\right\rangle=\sum_{a=1}^{M}\left|\frac{\partial \Psi}{\partial f_{a}}\right\rangle \delta f_{a}=0 \quad(M<N) .
$$

If the local bases $\left\{\left|\frac{\partial \Psi}{\partial f_{a}}\right\rangle\right\}_{a=1, M}$ are independent, the constraint (3) is equivalent to a set of constraints on the parameters as

$$
\delta f_{a}=0 \quad(a=1, \ldots, M) .
$$

When the variational freedom $\delta \Psi_{f}$ is located within the predetermined region of Eq. (1), the parameters $\left\{f_{a}\right\}_{a=1, M}$ will be functions of the primordial parameters $\left\{\alpha_{i}\right\}_{i=1, N}$. Then the explicit constraints (4) on $\left\{\delta f_{a}\right\}_{a=1, M}$ can be understood as implicit constraints on $\left\{\delta \alpha_{i}\right\}_{i=1, N}$ through the functions

$$
f_{a}=f_{a}\left(\alpha_{1}, \ldots, \alpha_{N}\right)=0 \quad(a=1, \ldots, M) .
$$

The equations (5) correspond to the holonomic constraints on dynamical variables in classical mechanics[25]. 
In order for the above equations to hold, we should introduce some requirements as follows. First we consider the requirement of the independence of the local bases $\left\{\left|\frac{\partial \Psi}{\partial f_{a}}\right\rangle\right\}_{a=1, M}$. The local bases of $\left\{f_{a}\right\}_{a=1, M}$ can be expanded as

$$
\left|\frac{\partial \Psi}{\partial f_{a}}\right\rangle=\left.\sum_{i=1}^{N}\left|\frac{\partial \Psi}{\partial \alpha_{i}}\right\rangle\left(\frac{\partial \alpha_{i}}{\partial f_{a}}\right)\right|_{0} \quad(a=1, \ldots, M),
$$

where $\left.\right|_{0}$ denotes calculations on the constraint surface, that is to apply Eq. (5) after differentiations. So, the independence of the local bases $\left\{\left|\frac{\partial \Psi}{\partial f_{a}}\right\rangle\right\}_{a=1, M}$ is equivalent to the requirement for the rectangular matrix $\left.\left(\frac{\partial \boldsymbol{\alpha}}{\partial \boldsymbol{f}}\right)\right|_{0}$ of dimensions $N \times M$ as

$$
\operatorname{Rank}\left[\left.\left(\frac{\boldsymbol{\partial} \boldsymbol{\alpha}}{\boldsymbol{\partial f}}\right)\right|_{0}\right]=\operatorname{Rank}\left[\left.\left(\frac{\partial \alpha_{i}}{\partial f_{a}}\right)\right|_{0}\right]=M .
$$

The equation (7) is the regularity condition of constraints and assures the nonsingularity of the static structure which is constructed with $\left\{\left|\frac{\partial \Psi}{\partial f_{a}}\right\rangle\right\}_{a=1, M}$. Next we consider the constraints (4) through Eq. (5) as

$$
\delta f_{a}=\left.\sum_{i=1}^{N}\left(\frac{\partial f_{a}}{\partial \alpha_{i}}\right)\right|_{0} \delta \alpha_{i}=0 .
$$

To obtain $M$-dimensional independent constraints (4), we should require again

$$
\operatorname{Rank}\left[\left.\left(\frac{\boldsymbol{\partial f}}{\boldsymbol{\partial \alpha}}\right)\right|_{0}\right]=\operatorname{Rank}\left[\left.\left(\frac{\partial f_{a}}{\partial \alpha_{i}}\right)\right|_{0}\right]=M,
$$

for the rectangular matrix of dimensions $M \times N$. The equation (9) is another regularity condition which has the same form as in classical-mechanics[20]. The regularity conditions (7) and (9) satisfy the reciprocal relation with each other as

$$
\left.\left.\left(\frac{\partial f}{\partial \alpha}\right)\right|_{0}\left(\frac{\partial \alpha}{\partial f}\right)\right|_{0}=E_{M}
$$

by the chain rule of partial differentiations as shown in Appendix A. The matrix $\boldsymbol{E}_{M}$ is an $M$-dimensional identity matrix. Not only in time-dependent but also in time-independent variational approaches, the regularity conditions (7) and (9) are required for the static nonsingularity of the constraint surface.

Finally, as a special form of constraints (5), we consider constraints for expectation values $[11,12]$ by some quantum operators $\left\{\hat{\xi}_{a}\right\}_{a=1, M}$ as

$$
\begin{aligned}
& \xi_{a}=\xi_{a}\left(\alpha_{1}, \ldots, \alpha_{N}\right)=\left\langle\Psi\left(\alpha_{1}, \ldots, \alpha_{N}\right)\left|\hat{\xi}_{a}\right| \Psi\left(\alpha_{1}, \ldots, \alpha_{N}\right)\right\rangle=0 \\
&(a=1, \ldots, M<N) .
\end{aligned}
$$


The constraints on the variational freedoms are written as

$$
\delta \xi_{a}=\left.\sum_{i=1}^{N}\left(\frac{\partial \xi_{a}}{\partial \alpha_{i}}\right)\right|_{0} \delta \alpha_{i}=\sum_{i=1}^{N}\left[\left.\left\langle\frac{\partial \Psi}{\partial \alpha_{i}}\left|\hat{\xi}_{a}\right| \Psi\right\rangle\right|_{0}+\left.\left\langle\Psi\left|\hat{\xi}_{a}\right| \frac{\partial \Psi}{\partial \alpha_{i}}\right\rangle\right|_{0}\right] \delta \alpha_{i}=0 .
$$

The regularity condition for the constraints (11) is

$$
\operatorname{Rank}\left[\left.\left(\frac{\boldsymbol{\partial} \boldsymbol{\xi}}{\boldsymbol{\partial \alpha}}\right)\right|_{0}\right]=\operatorname{Rank}\left[\left(\left.\left\langle\frac{\partial \Psi}{\partial \alpha_{i}}\left|\hat{\xi}_{a}\right| \Psi\right\rangle\right|_{0}+\left.\left\langle\Psi\left|\hat{\xi}_{a}\right| \frac{\partial \Psi}{\partial \alpha_{i}}\right\rangle\right|_{0}\right)\right]=M .
$$

So, if the wave function $\Psi$ satisfy a pseudo eigenvalue-equation on the constraint surface for one of the operators $\left\{\hat{\xi}_{a}\right\}_{a=1, M}$, namely for $\hat{\xi}_{b}$ as

$$
\left(\left.\left\langle\frac{\partial \Psi}{\partial \alpha_{i}}\left|\hat{\xi}_{b}\right| \Psi\right\rangle\right|_{0}+\left.\left\langle\Psi\left|\hat{\xi}_{b}\right| \frac{\partial \Psi}{\partial \alpha_{i}}\right\rangle\right|_{0}\right)=0 \quad(i=1, \ldots, N),
$$

the constraints (11) breaks the regularity condition (13) and can not construct any nonsingular constraint surface. 


\section{SECOND-CLASS CONDITION FOR DYNAMIC NONSINGULARITY OF CONSTRAINTS}

In this section we consider the second-class condition which assures nonsingularity of the dynamic structure of the constraint surface with a so-called symplectic metric[7].

\section{A. TDVP-EOM and generalized Poisson bracket (GPB)}

First we summarize the equation of motion of TDVP (TDVP-EOM)[7-10] for the parameters $\left\{\alpha_{i}(t)\right\}_{i=1, N}$. The real-valued Lagrangian of the TDVP is defined with a normalized wave function $\Psi$ as

$$
\begin{aligned}
L(\alpha, \dot{\alpha}) & =<\Psi(\alpha)|\hat{L}| \Psi(\alpha)>=<\Psi(\alpha)\left|\left(i \hbar \partial_{t}-\hat{H}\right)\right| \Psi(\alpha)> \\
& =i \hbar \sum_{i=1}^{N}\left\langle\Psi \mid \frac{\partial \Psi}{\partial \alpha_{i}}\right\rangle \dot{\alpha}_{i}-<\Psi|\hat{H}| \Psi>,
\end{aligned}
$$

where $\left\{\alpha_{i}(t)\right\}_{i=1, N}$ describe the time development of the wave function within the predetermined region of Eq. (1). If the wave function is not normalized, the normalization can be considered a posteriori since the norm of wave function is one of the constants of motion in the TDVP $[11,12]$. However, the operator $\hat{L}$ in Eq. (15) should be replaced by the Hermitian form as in Eq. (B1) of Appendix B. We obtain the EOM by the least-action principle

$$
\begin{aligned}
& \delta \int_{t_{1}}^{t_{2}} L(\alpha, \dot{\alpha}) d t \\
& =\sum_{i=1}^{N} \int_{t_{1}}^{t_{2}} \delta \alpha_{i}\left[\sum_{j=1}^{N} i \hbar\left(\left\langle\frac{\partial \Psi}{\partial \alpha_{i}} \mid \frac{\partial \Psi}{\partial \alpha_{j}}\right\rangle-\left\langle\frac{\partial \Psi}{\partial \alpha_{j}} \mid \frac{\partial \Psi}{\partial \alpha_{i}}\right\rangle\right) \dot{\alpha}_{j}-\frac{\partial<\Psi|\hat{H}| \Psi>}{\partial \alpha_{i}}\right] d t \\
& =0,
\end{aligned}
$$

with fixed boundary conditions as $\delta \alpha_{i}\left(t_{1}\right)=\delta \alpha_{i}\left(t_{2}\right)=0$. From Eq. (16), the TDVP-EOM for the parameters $\left\{\alpha_{i}(t)\right\}_{i=1, N}$ is obtained as

$$
\dot{\alpha}_{i}=\sum_{j=1}^{N}\left(\boldsymbol{\sigma}^{-1}\right)_{i j} \frac{\partial H}{\partial \alpha_{j}},
$$

where $H=<\Psi|\hat{H}| \Psi>$ and

$$
(\boldsymbol{\sigma})_{i j}=i \hbar\left(\left\langle\frac{\partial \Psi}{\partial \alpha_{i}} \mid \frac{\partial \Psi}{\partial \alpha_{j}}\right\rangle-\left\langle\frac{\partial \Psi}{\partial \alpha_{j}} \mid \frac{\partial \Psi}{\partial \alpha_{i}}\right\rangle\right)=i \hbar\left(\boldsymbol{S}-\boldsymbol{S}^{t}\right)_{i j} .
$$


The TDVP parameters $\left\{\alpha_{i}\right\}_{i=1, N}$ are not canonical variables in general. If the parameters are standard canonical variables as $\left\{q_{i}, p_{i}\right\}_{i=1, n}(N=2 n)$ in classical mechanics, the matrix $\boldsymbol{\sigma}^{-1}$ in Eq. (17) has a standard canonical form

$$
\boldsymbol{\sigma}^{-1}=\boldsymbol{J}=\left(\begin{array}{cc}
\mathbf{0} & \boldsymbol{E}_{n} \\
-\boldsymbol{E}_{n} & \mathbf{0}
\end{array}\right)=-\boldsymbol{J}^{-1}
$$

The real-valued anti-symmetric matrix $\boldsymbol{\sigma}$ is sometimes called as a symplectic metric of the dynamical space[7], although $\boldsymbol{\sigma}$ is not a "metric" in the exact meaning. When the matrix $\boldsymbol{\sigma}$ becomes noninvertible, we cannot continue to chase the time development of the system by Eq. (17). This singularity of $\boldsymbol{\sigma}$ is called as a symplectic singularity[22-24]. We here note that the nonsingularity of symplectic metric $\boldsymbol{\sigma}$ is not equal to that of Hermitian metric $\boldsymbol{S}$ in Eq. (2). Using the Hermitian property for $\boldsymbol{S}=\operatorname{Re}(\boldsymbol{S})+i \operatorname{Im}(\boldsymbol{S})$ in Eq. (18), we have

$$
\operatorname{Rank}[\boldsymbol{\sigma}]=\operatorname{Rank}[\operatorname{Im}(\boldsymbol{S})]
$$

Then the nonsingularity of the dynamic structure $\boldsymbol{\sigma}$ and that of the static structure $\boldsymbol{S}$ are not equivalent.

Finally we here introduce Generalized Poisson Bracket (GPB) [8, 25] as

$$
\{r, s\} \boldsymbol{\sigma}^{-1}=\sum_{i=1}^{N} \sum_{j=1}^{N} \frac{\partial r}{\partial \alpha_{i}}\left(\boldsymbol{\sigma}^{-1}\right)_{i j} \frac{\partial s}{\partial \alpha_{j}} .
$$

The GPB of the parameters $\left\{\alpha_{i}(t)\right\}_{i=1, N}$ by themselves is the inverse of the symplectic metric

$$
\{\alpha, \alpha\}_{\sigma^{-1}}=\sigma^{-1}
$$

The time development of any function $\Omega(\alpha)$ can be calculated by the GPB as

$$
\dot{\Omega}=\{\Omega, H\}_{\boldsymbol{\sigma}^{-1}}
$$

\section{B. TDVP-EOM with constraints}

We here apply constraints (5) for the dynamics of the parameters $\left\{\alpha_{i}(t)\right\}_{i=1, N}$. As Eq. (16), the least-action principle with the constraints is

$$
\sum_{i=1}^{N} \int_{t_{1}}^{t_{2}} \delta \alpha_{i}\left[\sum_{j=1}^{N}(\boldsymbol{\sigma})_{i j} \dot{\alpha}_{j}-\frac{\partial H(\alpha)}{\partial \alpha_{i}}-\sum_{a=1}^{M} \lambda_{a} \frac{\partial f_{a}(\alpha)}{\partial \alpha_{i}}\right] d t=0
$$


where Lagrange multipliers $\left\{\lambda_{a}\right\}_{a=1, M}$ are introduced. The TDVP-EOM with constraints are obtained as

$$
\dot{\alpha}_{i}=\sum_{j=1}^{N}\left(\boldsymbol{\sigma}^{-1}\right)_{i j} \frac{\partial K}{\partial \alpha_{j}},
$$

where an extended Hamiltonian[14, 15] is defined as

$$
K(\alpha)=H(\alpha)+\sum_{a=1}^{M} \lambda_{a} f_{a}(\alpha) .
$$

The constraints should be preserved in time along the trajectory of $\left\{\alpha_{i}(t)\right\}_{i=1, N}$. This is the consistency condition in Dirac's constrained classical-mechanics[14, 15]. By using the EOM (25), the consistency conditions for the constraints (5) are written as

$$
\dot{f}_{a}=\left\{f_{a}, H\right\} \boldsymbol{\sigma}^{-1}+\sum_{b=1}^{M}\left\{f_{a}, f_{b}\right\}_{\boldsymbol{\sigma}^{-1}} \lambda_{b}=0 .
$$

If we define a column vector $(\boldsymbol{h})_{a}=\left\{f_{a}, H\right\}_{\boldsymbol{\sigma}^{-1}}$, the inhomogeneous linear equations (27) are rewritten in a matrix form as

$$
h+\{f, f\}_{\sigma^{-1}} \lambda=0 .
$$

The Lagrange multipliers are obtained as

$$
\lambda=-\left(\{f, f\}_{\sigma^{-1}}\right)^{-1} h
$$

\section{Second-class condition of constraints}

In order to determine the Lagrange multipliers uniquely by Eq. (29), we should assume the nonsingularity of the Poisson matrix $\{\boldsymbol{f}, \boldsymbol{f}\}_{\boldsymbol{\sigma}^{-1}}$. So, the constraints (5) are required to satisfy another condition on the constraint surface as

$$
\operatorname{Rank}\left[\{\boldsymbol{f}, \boldsymbol{f}\}_{\boldsymbol{\sigma}^{-1}}\right]=M
$$

The constraints which satisfy Eq. (30) have been classified to second-class constraints[14, 15]. So we call here Eq. (30) as the second-class condition for the constraints.

The GPB of the constraints is written by the parameters $\left\{\alpha_{i}(t)\right\}_{i=1, N}$ as

$$
\{f, f\}_{\sigma^{-1}}=\left.\left.\left(\frac{\partial f}{\partial \alpha}\right)\right|_{0} \sigma^{-1}\left(\frac{\partial f}{\partial \alpha}\right)^{t}\right|_{0} .
$$


Then if the symplectic metric $\boldsymbol{\sigma}$ is nonsingular, we have

$$
\operatorname{Rank}\left[\{\boldsymbol{f}, \boldsymbol{f}\}_{\boldsymbol{\sigma}^{-1}}\right] \leq \operatorname{Rank}\left[\left.\left(\frac{\boldsymbol{\partial} \boldsymbol{f}}{\boldsymbol{\partial \alpha}}\right)\right|_{0}\right] \leq M .
$$

So the regularity condition (9) is only a necessary condition for the second-class condition (30). 


\section{ANALYTIC WAVE FUNCTIONS FOR COMPLEX TDVP-PARAMETERS}

The TDVP with complex parameters is just a special case of the real parameters. The complex parametrization, however, has some simple features for the second-class condition and we treat it here separately.

\section{A. TDVP-EOM and complex generalized Poisson bracket (CGPB)}

We here adopt analytic wave functions[8] for complex TDVP-parameters $\left\{z_{i}, z_{i}^{*}\right\}_{i=1, n}$ with $N=2 n$ as

$$
\Psi(z)=\Psi\left(z_{1}, z_{2}, \ldots, z_{n}\right), \quad \frac{\partial \Psi(z)}{\partial z_{i}^{*}}=0 \quad(i=1, \ldots, n),
$$

to keep the variational independency or the duality between $\delta \Psi$ and $\delta \Psi^{*}$. As shown in Appendix B, if the Gramian matrix is defined as

$$
(\boldsymbol{C})_{i j}=\left\langle\frac{\partial \Psi}{\partial z_{i}} \mid \frac{\partial \Psi}{\partial z_{j}}\right\rangle
$$

the TDVP-EOM (B3) is written in a matrix form as

$$
\left(\begin{array}{c}
\dot{z} \\
\dot{z^{*}}
\end{array}\right)=\frac{1}{i \hbar}\left(\begin{array}{cc}
0 & \boldsymbol{C}^{-1} \\
-\left(\boldsymbol{C}^{-1}\right)^{t} & \mathbf{0}
\end{array}\right)\left(\begin{array}{c}
\frac{\partial \boldsymbol{H}}{\partial \boldsymbol{z}} \\
\frac{\partial \boldsymbol{H}}{\partial \boldsymbol{z}^{*}}
\end{array}\right)=\boldsymbol{\sigma}^{-1}\left(\begin{array}{c}
\frac{\partial \boldsymbol{H}}{\partial \boldsymbol{z}} \\
\frac{\partial \boldsymbol{H}}{\partial \boldsymbol{z}^{*}}
\end{array}\right) .
$$

In this case, we have

$$
|\boldsymbol{\sigma}|=(-1)^{n} \hbar^{2 n}|\boldsymbol{C}|^{2},
$$

for the symplectic metric

$$
\boldsymbol{\sigma}=i \hbar\left(\begin{array}{cc}
0 & -\boldsymbol{C}^{t} \\
\boldsymbol{C} & \mathbf{0}
\end{array}\right)
$$

Therefore, differently from the case of the real parameters as in Eq. (20), the nonsingularity of the dynamic structure $\boldsymbol{\sigma}$ and that of the static structure $\boldsymbol{C}$ become equivalent in the complex parametrization.

The GPB (21) is reduced to the Complex Generalized Poisson Bracket (CGPB) [8, 12] as

$$
\{r, s\}_{\boldsymbol{\sigma}^{-1}}=\frac{1}{i \hbar} \sum_{i=1}^{n} \sum_{j=1}^{n}\left[\frac{\partial r}{\partial z_{i}}\left(\boldsymbol{C}^{-1}\right)_{i j} \frac{\partial s}{\partial z_{j}^{*}}-\frac{\partial r}{\partial z_{i}^{*}}\left[\left(\boldsymbol{C}^{-1}\right)^{t}\right]_{i j} \frac{\partial s}{\partial z_{j}}\right]=\{r, s\}_{C G P B} .
$$

The time development of any function $\Omega\left(z, z^{*}\right)$ can be calculated as

$$
\dot{\Omega}=\{\Omega, H\}_{C G P B}
$$




\section{B. Regularity condition of constraints}

We here introduce constraints for the complex parameters $\left\{z_{i}, z_{i}^{*}\right\}_{i=1, n}$. In order keep the duality of $\delta \Psi$ and $\delta \Psi^{*}$, the constraints are also taken to be analytic as

$$
u_{a}\left(z_{1}, \ldots, z_{n}\right)=0, \quad u_{a}^{*}\left(z_{1}, \ldots, z_{n}\right)=0 \quad(a=1, \ldots, m<n) .
$$

Variational freedoms $\left\{\delta z_{i}, \delta z_{i}^{*}\right\}_{i=1, n}$ are constrained implicitly as

$$
\delta u_{a}=\left.\sum_{i=1}^{n}\left(\frac{\partial u_{a}}{\partial z_{i}}\right)\right|_{0} \delta z_{i}=0, \quad \delta u_{a}^{*}=\left.\sum_{i=1}^{n}\left(\frac{\partial u_{a}^{*}}{\partial z_{i}^{*}}\right)\right|_{0} \delta z_{i}^{*}=0 .
$$

As the real parameters in Eqs. (9) and (7), the regularity conditions of the constraints (40) are

$$
\operatorname{Rank}\left[\left.\left(\frac{\boldsymbol{\partial u}}{\boldsymbol{\partial} \boldsymbol{z}}\right)\right|_{0}\right]=\operatorname{Rank}\left[\left.\left(\frac{\boldsymbol{\partial u}}{\boldsymbol{\partial} \boldsymbol{z}}\right)^{\dagger}\right|_{0}\right]=m
$$

and

$$
\operatorname{Rank}\left[\left.\left(\frac{\boldsymbol{\partial} \boldsymbol{z}}{\boldsymbol{\partial u}}\right)\right|_{0}\right]=\operatorname{Rank}\left[\left.\left(\frac{\boldsymbol{\partial} \boldsymbol{z}}{\boldsymbol{\partial u}}\right)^{\dagger}\right|_{0}\right]=m
$$

respectively.

\section{Second-class condition of constraints}

As shown in Appendix B, the constraints (40) are considered in the TDVP with Lagrange multipliers. In order to determine the Lagrange multipliers uniquely from the consistency conditions of the constraints $u_{a}(z)=0$ as in Eq. (B8), we should require the second-class condition on the constraint surface as

$$
\operatorname{Rank}\left[\left\{\boldsymbol{u}, \boldsymbol{u}^{*}\right\}_{C G P B}\right]=m .
$$

Moreover, if we can assume the orthogonality between local bases as in Appendix C, we have

$$
\left\{\boldsymbol{u}, \boldsymbol{u}^{*}\right\}_{C G P B}=\left.\left.\frac{1}{i \hbar}\left(\frac{\partial \boldsymbol{u}}{\partial \boldsymbol{z}}\right)\right|_{0} \boldsymbol{C}^{-1}\left(\frac{\partial \boldsymbol{u}}{\partial \boldsymbol{z}}\right)^{\dagger}\right|_{0}=\frac{1}{i \hbar}\left\langle\frac{\partial \Psi}{\partial \boldsymbol{u}} \mid \frac{\partial \Psi}{\partial \boldsymbol{u}}\right\rangle^{-1} .
$$

Then, differently again from the case of the real parameters in Eq. (32), the regularity condition (42) and the second-class condition (44) become equivalent. Since $\operatorname{Rank}\left[\left\{\boldsymbol{u}, \boldsymbol{u}^{*}\right\}_{C G P B}\right]=\operatorname{Rank}\left[\left\{\boldsymbol{u}^{*}, \boldsymbol{u}\right\}_{C G P B}\right]$, the second-class condition for the other constraints $u_{a}^{*}(z)=0$ in Eq. (40) is also equivalent to the regularity condition (42). 


\section{Constraints for expectation values}

In this section, we show another special feature in the complex TDVP parameters. As in Eq. (11), we consider constraints of expectation values for some quantum operators

$$
\begin{array}{r}
\xi_{a}=\xi_{a}\left(z_{1}, \ldots, z_{n}, z_{1}^{*}, \ldots, z_{n}^{*}\right)=\left\langle\Psi\left(z_{1}, \ldots, z_{n}\right)\left|\hat{\xi}_{a}\right| \Psi\left(z_{1}, \ldots, z_{n}\right)\right\rangle=0 \\
(a=1, \ldots, m<n) .
\end{array}
$$

The second-class condition for the expectation values (46) can be examined simply by the algebra of corresponding quantum operators without any calculation of the expectation values as follows. If the local bases $\left\{\left|\frac{\partial \Psi}{\partial z_{i}}\right\rangle\right\}_{i=1, n}$ are complete locally, we have

$$
\sum_{i=1}^{n} \sum_{j=1}^{n}\left|\frac{\partial \Psi}{\partial z_{i}}\right\rangle\left(\boldsymbol{C}^{-1}\right)_{i j}\left\langle\frac{\partial \Psi}{\partial z_{j}}\right|=\hat{1} \text {. }
$$

In this case, the CGPB for the constraints becomes the expectation value of the commutator of corresponding operators [12] as

$$
\begin{aligned}
& \left\{\xi_{a}, \xi_{b}\right\}_{C G P B} \\
& =\frac{1}{i \hbar} \sum_{i=1}^{n} \sum_{j=1}^{n}\left[\frac{\partial<\Psi\left|\hat{\xi}_{a}\right| \Psi>}{\partial z_{i}}\left(\boldsymbol{C}^{-1}\right)_{i j} \frac{\partial<\Psi\left|\hat{\xi}_{b}\right| \Psi>}{\partial z_{j}^{*}}\right. \\
& \left.-\frac{\partial<\Psi\left|\hat{\xi}_{a}\right| \Psi>}{\partial z_{i}^{*}}\left[\left(\boldsymbol{C}^{-1}\right)^{t}\right]_{i j} \frac{\partial<\Psi\left|\hat{\xi}_{b}\right| \Psi>}{\partial z_{j}}\right] \\
& =\frac{1}{i \hbar}\left[\left\langle\Psi\left|\hat{\xi}_{a}\left(\sum_{i=1}^{n} \sum_{j=1}^{n}\left|\frac{\partial \Psi}{\partial z_{i}}\right\rangle\left(\boldsymbol{C}^{-1}\right)_{i j}\left\langle\frac{\partial \Psi}{\partial z_{j}}\right|\right) \hat{\xi}_{b}\right| \Psi\right\rangle\right. \\
& \left.-\left\langle\Psi\left|\hat{\xi}_{b}\left(\sum_{i=1}^{n} \sum_{j=1}^{n}\left|\frac{\partial \Psi}{\partial z_{i}}\right\rangle\left(\boldsymbol{C}^{-1}\right)_{i j}\left\langle\frac{\partial \Psi}{\partial z_{j}}\right|\right) \hat{\xi}_{a}\right| \Psi\right\rangle\right] \\
& =\frac{1}{i \hbar}<\Psi\left|\left[\hat{\xi}_{a}, \hat{\xi}_{b}\right]\right| \Psi>.
\end{aligned}
$$

Then commutable operators $\left[\hat{\xi}_{a}, \hat{\xi}_{b}\right]=0$ always lead to singular constraints. We note, however, that Eq. (48) is true if and only if the local completeness condition (47) holds. Then, even if operators are commutable, they will construct nonsingular constraint surface for approximate wave functions which do not have the local completeness (47). 


\section{EXAMPLES OF SECOND-CLASS CONDITIONS}

\section{A. Augmentation of constraints}

We here consider the case that new constraints $\left\{g_{b}=0\right\}_{b=1, M_{2}}$ are added to the existing nonsingular constraints $\left\{f_{a}=0\right\}_{a=1, M_{1}}$ as

$$
\begin{array}{ll}
f_{a}=f_{a}\left(\alpha_{1}, \ldots, \alpha_{N}\right)=0 & \left(a=1, \ldots, M_{1}\right), \\
g_{b}=g_{b}\left(\alpha_{1}, \ldots, \alpha_{N}\right)=0 & \left(b=1, \ldots, M_{2}\right) .
\end{array}
$$

The dimension of the extended frozen-space will be $M=\left(M_{1}+M_{2}\right)<N$ as

$$
\boldsymbol{h}=(\boldsymbol{f}, \boldsymbol{g})=\left(f_{1}, f_{2}, \ldots, f_{M_{1}}, g_{1}, g_{2}, \ldots, g_{M_{2}}\right)=\mathbf{0} .
$$

The GPB matrix of the total constraints to examine the second-class condition is written as

$$
\{h, h\}_{\sigma^{-1}}=\left(\begin{array}{ll}
\{f, f\}_{\sigma^{-1}} & \{f, g\}_{\sigma^{-1}} \\
\{g, f\}_{\sigma^{-1}} & \{g, g\}_{\sigma^{-1}}
\end{array}\right) .
$$

We here use an equation of factorization for a matrix of dimensions $(K+L) \times(K+L)$ with partial matrices $\boldsymbol{A}_{K \times K}, \boldsymbol{B}_{K \times L}, \boldsymbol{C}_{L \times K}$, and $\boldsymbol{D}_{L \times L}$ as

$$
\left(\begin{array}{ll}
\boldsymbol{A} & \boldsymbol{B} \\
\boldsymbol{C} & \boldsymbol{D}
\end{array}\right)=\left(\begin{array}{cc}
\boldsymbol{E}_{K} & \mathbf{0} \\
\boldsymbol{C} \boldsymbol{A}^{-1} & \boldsymbol{E}_{L}
\end{array}\right)\left(\begin{array}{cc}
\boldsymbol{A} & \mathbf{0} \\
\mathbf{0} & \boldsymbol{D}-\boldsymbol{C} \boldsymbol{A}^{-1} \boldsymbol{B}
\end{array}\right)\left(\begin{array}{cc}
\boldsymbol{E}_{K} & \boldsymbol{A}^{-1} \boldsymbol{B} \\
\mathbf{0} & \boldsymbol{E}_{L}
\end{array}\right) .
$$

This equation is proven easily by straightforward calculation[26]. Using Eq. (53), we can decompose the determinant of the matrix (52) as

$$
\begin{aligned}
\left|\{h, h\}_{\sigma^{-1}}\right| & =\left|\begin{array}{cc}
\{f, f\}_{\sigma^{-1}} & \{f, g\}_{\sigma^{-1}} \\
\{g, f\}_{\sigma^{-1}} & \{g, g\}_{\sigma^{-1}}
\end{array}\right| \\
& =\left|\{f, f\}_{\sigma^{-1}}\right| \times\left|\{g, g\}_{\sigma^{-1}}-\{g, f\}_{\sigma^{-1}}\left(\{f, f\}_{\sigma^{-1}}\right)^{-1}\{f, g\}_{\sigma^{-1}}\right|(5)
\end{aligned}
$$

By using Eq. (54), we can analyze the nonsingularity of the total constraints (51). If we assume the existing constraints $\left\{f_{a}=0\right\}_{a=1, M_{1}}$ are nonsingular in the extended frozen-space, the singularity of the GPB $\{\boldsymbol{h}, \boldsymbol{h}\}_{\boldsymbol{\sigma}^{-1}}$ is caused by

$$
\left|\{\boldsymbol{g}, \boldsymbol{g}\}_{\sigma^{-1}}-\{\boldsymbol{g}, \boldsymbol{f}\}_{\boldsymbol{\sigma}^{-1}}\left(\{\boldsymbol{f}, \boldsymbol{f}\}_{\boldsymbol{\sigma}^{-1}}\right)^{-1}\{\boldsymbol{f}, \boldsymbol{g}\}_{\boldsymbol{\sigma}^{-1}}\right|=0
$$

The matrix in the determinant (55) is constructed by the components of $\boldsymbol{g}$, which are orthogonalized to $f$ by Schmidt-like method with the symplectic metric $\{f, f\}_{\sigma^{-1}}$. So 
there are two possibilities for Eq. (55) to hold. One is that the added constraints $\boldsymbol{g}$ do not have new components enough to construct the extended frozen-space. The other is the case that $\{\boldsymbol{f}, \boldsymbol{g}\}_{\boldsymbol{\sigma}^{-1}}=\mathbf{0}$ and $\left|\{\boldsymbol{g}, \boldsymbol{g}\}_{\boldsymbol{\sigma}^{-1}}\right|=0$ at the same time. This means that $\boldsymbol{g}$ is Poisson commutable to $\boldsymbol{f}$ and moreover singular by itself.

\section{B. Constraints for moments}

We consider moments as an example of the expectation values in Eq. (11).

Before the expectation values in quantum mechanics, we constrain the powers of the TDVP parameters directly as in classical mechanics. If the powers of a variable $\omega(\alpha)=$ $\omega\left(\alpha_{1}, \ldots, \alpha_{N}\right)$ are constrained directly as $f_{r}(\alpha)=\omega^{r}(\alpha)-\mu_{r}=0$ and $f_{s}(\alpha)=\omega^{s}(\alpha)-\mu_{s}=0$, we have their GPB as

$$
\begin{aligned}
\left\{f_{r}, f_{s}\right\} \boldsymbol{\sigma}^{-1} & =\sum_{i=1}^{N} \sum_{j=1}^{N} \frac{\partial\left(\omega^{r}-\mu_{r}\right)}{\partial \alpha_{i}}\left(\boldsymbol{\sigma}^{-1}\right)_{i j} \frac{\partial\left(\omega^{s}-\mu_{s}\right)}{\partial \alpha_{j}} \\
& =r s \omega^{r+s-2}\{\omega, \omega\}_{\boldsymbol{\sigma}^{-1}}=0 .
\end{aligned}
$$

So the constraints $f_{r}$ and $f_{s}$ can not construct any nonsingular constraint surface as in classical mechanics. We note that these constraints break also the regularity condition (9), since they are degenerate as $\left(\frac{\partial f_{r}}{\partial \alpha_{i}}\right)=\left[\omega^{r-s}\left(\frac{r}{s}\right)\right]\left(\frac{\partial f_{s}}{\partial \alpha_{i}}\right)$.

Next, as the constraints for expectation values, we constrain the moments of $r$-th power of a Hermitian operator $\hat{\omega}$ as

$$
f_{r}(\alpha)=<\Psi(\alpha)\left|\hat{f}_{r}\right| \Psi(\alpha)>=<\Psi(\alpha)\left|\hat{\omega}^{r}-\mu_{r}\right| \Psi(\alpha)>=0 \quad(r=1,2, \ldots)
$$

The constants should satisfy $\mu_{2} \geq\left(\mu_{1}\right)^{2} \geq 0$ and so on, as shown generally in Appendix D. The expectation values have usually the variance as

$$
<\Psi\left|\hat{\omega}^{r+s}\right| \Psi>\neq<\Psi\left|\hat{\omega}^{r}\right| \Psi><\Psi\left|\hat{\omega}^{s}\right| \Psi>.
$$

So, we have

$$
\begin{aligned}
& \left\{f_{r}, f_{s}\right\}_{\boldsymbol{\sigma}^{-1}}=\left\{<\Psi\left|\hat{\omega}^{r}-\mu_{r}\right| \Psi>,<\Psi\left|\hat{\omega}^{s}-\mu_{s}\right| \Psi>\right\}_{\boldsymbol{\sigma}^{-1}} \\
& \neq r s<\Psi|\hat{\omega}| \Psi>^{r+s-2}\left(\sum_{i=1}^{N} \sum_{j=1}^{N} \frac{\partial<\Psi|\hat{\omega}| \Psi>}{\partial \alpha_{i}}\left(\boldsymbol{\sigma}^{-1}\right)_{i j} \frac{\partial<\Psi|\hat{\omega}| \Psi>}{\partial \alpha_{j}}\right)=0 .
\end{aligned}
$$


Then the constraints for moments can be nonsingular. If we employ the analytic wave function (33), however, we have

$$
\begin{aligned}
\left\{f_{r}, f_{s}\right\}_{C G P B} & =\left\{<\Psi\left|\hat{\omega}^{r}-\mu_{r}\right| \Psi>,<\Psi\left|\hat{\omega}^{s}-\mu_{s}\right| \Psi>\right\}_{C G P B} \\
& =\frac{1}{i \hbar}<\Psi\left|\left[\hat{\omega}^{r}, \hat{\omega}^{s}\right]\right| \Psi>=0,
\end{aligned}
$$

through Eq. (48). So the constraints become singular again as in Eq. (56) or in classical mechanics.

\section{Singularity of TDVP-EOM}

As the last example of the second-class condition, we discuss the symplectic singularity of the TDVP-EOM[23, 24]. In the EOM (17), if the matrix $\boldsymbol{\sigma}$ becomes singular, we cannot continue to chase the time development of the system. As in classical mechanics[22], the symplectic singularity in the TDVP-EOM is shown to be a local breakdown of the secondclass condition in an extended canonical phase-space as follows.

For the TDVP-EOM (17), we consider the Legendre transformations of dynamical variables to obtain the EOM in a canonical phase-space as usually in classical mechanics. However, the TDVP Lagrangian $L(\alpha, \dot{\alpha})(15)$ is linear in $\dot{\alpha}_{i}$, and we cannot introduce corresponding variables by $\beta_{i}=\frac{\partial L}{\partial \dot{\alpha}_{i}}$ because the Jacobian matrix of the transformation is always singular as $\left|\frac{\partial \beta_{i}}{\partial \dot{\alpha}_{j}}\right|=\left|\frac{\partial^{2} L}{\partial \dot{\alpha}_{i} \partial \dot{\alpha}_{j}}\right|=0$. So, we here construct $2 n$-dimensional extended phase-space $\left\{\alpha_{i}, \beta_{i}\right\}_{i=1, n}$ by adding the "momentum" variables $\left\{\beta_{i}(t)\right\}_{i=1, n}$ with $n$ constraints as

$$
f_{i}(\alpha, \beta)=\beta_{i}-\frac{\partial L(\alpha, \dot{\alpha})}{\partial \dot{\alpha}_{i}}=\beta_{i}-i \hbar\left\langle\Psi(\alpha) \mid \frac{\partial \Psi(\alpha)}{\partial \alpha_{i}}\right\rangle=0 \quad(i=1, \ldots, n) .
$$

The action of the TDVP Lagrangian (15) is written as

$$
J_{T D V P}=\int_{t_{1}}^{t_{2}}\left[\sum_{i=1}^{n} \beta_{i} \dot{\alpha}_{i}-H(\alpha)\right] d t .
$$

So, by using Lagrange multipliers $\left\{\lambda_{i}\right\}_{i=1, n}$ as in Eq. (24), the TDVP with constraints (61) leads to

$$
\sum_{i=1}^{n} \int_{t_{1}}^{t_{2}}\left[\left(\dot{\alpha}_{i}-\frac{\partial H}{\partial \beta_{i}}-\sum_{j=1}^{n} \lambda_{j} \frac{\partial f_{j}}{\partial \beta_{i}}\right) \delta \beta_{i}-\left(\dot{\beta}_{i}+\frac{\partial H}{\partial \alpha_{i}}+\sum_{j=1}^{n} \lambda_{j} \frac{\partial f_{j}}{\partial \alpha_{i}}\right) \delta \alpha_{i}\right] d t=0 .
$$

If an extended TDVP Hamiltonian $K(\alpha, \beta)$ is defined as

$$
K(\alpha, \beta)=H(\alpha)+\sum_{j=1}^{n} \lambda_{j} f_{j}(\alpha, \beta)
$$


the EOM is written as

$$
\dot{\alpha}_{i}=\frac{\partial K}{\partial \beta_{i}}, \quad \dot{\beta}_{i}=-\frac{\partial K}{\partial \alpha_{i}} .
$$

So $\left\{\alpha_{i}, \beta_{i}\right\}_{i=1, n}$ become canonical variables and their Poisson bracket matrix has the $2 n$ dimensional canonical form $\boldsymbol{J}$ as in Eq. (19).

The Lagrange multipliers are determined by the consistency conditions for the constraints as Eq. (27)

$$
\sum_{j=1}^{n}\left\{f_{i}, f_{j}\right\}_{\boldsymbol{J}} \lambda_{j}=-\left\{f_{i}, H\right\}_{\boldsymbol{J}}
$$

We can show that the Poisson bracket matrix for the constraints (61) is reduced to the $n$-dimensional symplectic metric $\boldsymbol{\sigma}$ as in classical mechanics[22],

$$
\begin{aligned}
\left\{f_{i}, f_{j}\right\}_{\boldsymbol{J}} & =\sum_{k=1}^{n}\left[\frac{\partial f_{i}}{\partial \alpha_{k}} \frac{\partial f_{j}}{\partial \beta_{k}}-\frac{\partial f_{j}}{\partial \alpha_{k}} \frac{\partial f_{i}}{\partial \beta_{k}}\right] \\
& =i \hbar\left[\left\langle\frac{\partial \Psi}{\partial \alpha_{i}} \mid \frac{\partial \Psi}{\partial \alpha_{j}}\right\rangle-\left\langle\frac{\partial \Psi}{\partial \alpha_{j}} \mid \frac{\partial \Psi}{\partial \alpha_{i}}\right\rangle\right]=(\boldsymbol{\sigma})_{i j} .
\end{aligned}
$$

So, the symplectic singularity of $\boldsymbol{\sigma}$ in the EOM (17) can be understood as a local breakdown of the second-class condition for the constraints (61) in the extended canonical phase-space.

Finally we obtain the TDVP-EOM in the extended canonical phase-space explicitly as

$$
\dot{\alpha}_{i}=\lambda_{i}
$$

and

$$
\dot{\beta}_{i}=\frac{d}{d t}\left[i \hbar\left\langle\Psi \mid \frac{\partial \Psi}{\partial \alpha_{i}}\right\rangle\right]
$$

It is easy to show that the EOM (68) is reduced to Eq. (17) by using Eqs. (66), (67), and

$$
\left\{f_{i}, H\right\}_{\boldsymbol{J}}=-\frac{\partial H}{\partial \alpha_{i}}
$$

The other EOM (69) simply means the consistency conditions of constraints hold as $\dot{f}_{i}=0$.

\section{Numerical example}

We show a numerical example of the symplectic singularity of the TDVP-EOM. We consider a normalized wave function with real parameters $\theta(t)$ and $\eta(t)$ as

$$
\Psi(t ; x)=\frac{1}{\sqrt{\theta^{2}(t)+\eta^{2}(t)}}\left[\eta(t) e^{i \theta(t)} \chi_{1}(x)+\theta(t) e^{i \eta(t)} \chi_{2}(x)\right],
$$


where $\chi_{1}(x)$ and $\chi_{2}(x)$ are orthonormalized real-valued bases. By denoting $H_{i j}=<$ $\chi_{i}|\hat{H}| \chi_{j}>$, the TDVP Lagrangian (15) is calculated as

$$
L(\theta, \eta, \dot{\theta}, \dot{\eta})=-\hbar\left[\frac{\dot{\theta} \eta^{2}+\dot{\eta} \theta^{2}}{\theta^{2}+\eta^{2}}\right]-\left[\frac{\eta^{2} H_{11}+\theta^{2} H_{22}+2 \theta \eta \cos (\theta-\eta) H_{12}}{\theta^{2}+\eta^{2}}\right] .
$$

To construct the 4-dimensional extended canonical phase-space with $\left\{\theta, \beta_{\theta}, \eta, \beta_{\eta}\right\}$, constraints are introduced as

$$
f_{\theta}=\beta_{\theta}+\hbar\left(\theta^{2}+\eta^{2}\right)^{-1} \eta^{2}=0, \quad f_{\eta}=\beta_{\eta}+\hbar\left(\theta^{2}+\eta^{2}\right)^{-1} \theta^{2}=0 .
$$

Based on Eq. (67), the symplectic singularity of the TDVP-EOM (17) is examined by

$$
\left|\boldsymbol{\sigma}^{-1}\right|=\left|\left(\begin{array}{cc}
0 & \sigma_{\theta \eta} \\
\sigma_{\eta \theta} & 0
\end{array}\right)^{-1}\right|=\frac{1}{\left(\sigma_{\theta \eta}\right)^{2}}=\frac{1}{\left(\left\{f_{\theta}, f_{\eta}\right\}_{\boldsymbol{J}}\right)^{2}}=\left[\frac{1}{\hbar} \frac{\left(\theta^{2}+\eta^{2}\right)^{2}}{2 \theta \eta(\theta-\eta)}\right]^{2} .
$$

So the trajectories become singular at the points $\theta=0, \eta=0$, or $\theta=\eta$, where the constraints $f_{\theta}$ and $f_{\eta}$ will also break the second-class condition as $\left\{f_{\theta}, f_{\eta}\right\}_{\boldsymbol{J}}=0$ in the 4-dimensional canonical phase-space. The EOM in the 4-dimensional canonical phase-space is written explicitly as

$$
\begin{aligned}
\dot{\theta}= & -\frac{1}{\hbar}\left[\frac{1}{\eta(\theta-\eta)}\right] \\
& \times\left\{\theta \eta\left(H_{11}-H_{22}\right)+\left[\left(\theta^{2}-\eta^{2}\right) \cos (\theta-\eta)+\eta\left(\theta^{2}+\eta^{2}\right) \sin (\theta-\eta)\right] H_{12}\right\}, \\
\dot{\eta}= & -\frac{1}{\hbar}\left[\frac{1}{\theta(\theta-\eta)}\right] \\
& \times\left\{\theta \eta\left(H_{11}-H_{22}\right)+\left[\left(\theta^{2}-\eta^{2}\right) \cos (\theta-\eta)+\theta\left(\theta^{2}+\eta^{2}\right) \sin (\theta-\eta)\right] H_{12}\right\},
\end{aligned}
$$

and

$$
\begin{gathered}
\dot{\beta}_{1}=2 \theta \eta\left(\theta^{2}+\eta^{2}\right)^{-1} \sin (\theta-\eta) H_{12}, \\
\dot{\beta}_{2}=-\dot{\beta}_{1} .
\end{gathered}
$$

Numerically obtained trajectories are shown in Figure 1 for $\{\theta(t), \eta(t)\}$, and Figure 2 for $\left\{\beta_{\theta}(t), \beta_{\eta}(t)\right\}$, respectively. We have used the Hamiltonian parameters as $\left(H_{11}-H_{22}\right) / \hbar=$ $-2.0, H_{12} / \hbar=1.0$. Initial conditions for eight trajectories are $\left(\theta_{0}, \eta_{0}\right)=( \pm 0.5, \pm 0.25)$, and $( \pm 0.25, \pm 0.5)$. As shown in Figure 1, these eight trajectories, which do not approach to 
the origin along $\theta=0, \eta=0$, or $\theta=\eta$, go through the origin without any singularities. The continuity of these trajectories at the origin is proven in Appendix E. However, the trajectory, which starts from the initial condition $\left(\theta_{0}, \eta_{0}\right)=(0.25,0.5)$ or $(-0.25,-0.5)$, will sink into the singularity of $\eta=\theta$ as time goes on. The trajectories of $\left\{\beta_{\theta}, \beta_{\eta}\right\}$ are obtained directly by the constraints (73) as

$$
-\hbar \leq \beta_{\theta}(t) \leq 0, \quad-\hbar \leq \beta_{\eta}(t) \leq 0, \quad \beta_{\theta}(t)+\beta_{\eta}(t)=-\hbar
$$

The symplectic singularities are located at $\left(\beta_{\theta} / \hbar, \beta_{\eta} / \hbar\right)=(-1.0,0),(0,-1.0)$, and $(-0.5,-0.5)$ in Figure 2. 


\section{SUMMARY}

In this work we have investigated two conditions for the constraints in the TDVP. The regularity condition assures the static nonsingularity of the constraint surface not only in time-dependent but also in time-independent variational approaches. The constraint on the wave function which is the essential dynamical variable in quantum mechanics is connected to the nonsingular constraints on variational parameters through the regularity condition. The other condition is the second-class condition of the constraints which assures the dynamic nonsingularity of the constraint surface with a symplectic metric. Especially for analytic wave functions for complex TDVP-parameters, these two conditions become equivalent. For constraints of expectation values in complex TDVP-parameters, the second-class condition is reduced to the non-commutability of the corresponding quantum operators. As one of examples of the second-class condition, the symplectic singularity of the TDVP-EOM is shown to be a local breakdown of the second-class condition in the extended canonical phase-space. 


\section{Appendices}

\section{Appendix A: TRANSFORMATION OF REAL PARAMETERS}

We consider the nonsingular transformation from the parameters $\left\{\alpha_{i}\right\}_{i=1, N}$ to the parameters $\left\{f_{a}\right\}_{a=1, M}$ which will be frozen and their orthogonal complements $\left\{g_{b}\right\}_{b=1, N-M}$ as

$$
f_{a}=f_{a}\left(\alpha_{1}, \ldots, \alpha_{N}\right)(a=1, \ldots, M<N), g_{b}=g_{b}\left(\alpha_{1}, \ldots, \alpha_{N}\right)(b=1, \ldots, N-M \backslash \mathrm{A} 1)
$$

The variational freedoms are transformed as

$$
\delta f_{a}=\sum_{i=1}^{N}\left(\frac{\partial f_{a}}{\partial \alpha_{i}}\right) \delta \alpha_{i}, \quad \delta g_{b}=\sum_{i=1}^{N}\left(\frac{\partial g_{b}}{\partial \alpha_{i}}\right) \delta \alpha_{i}
$$

or in a matrix form

$$
\left(\begin{array}{l}
\delta f \\
\delta g
\end{array}\right)=\left(\begin{array}{l}
\frac{\partial f}{\partial \alpha} \\
\frac{\partial g}{\partial \alpha}
\end{array}\right) \delta \alpha
$$

When the Jacobian matrix is nonsingular, we have

$$
E_{N}=\left(\begin{array}{l}
\frac{\partial f}{\partial \alpha} \\
\frac{\partial g}{\partial \alpha}
\end{array}\right)\left(\begin{array}{ll}
\frac{\partial \alpha}{\partial f} & \frac{\partial \alpha}{\partial g}
\end{array}\right)=\left(\begin{array}{ll}
\frac{\partial f}{\partial \alpha} \frac{\partial \alpha}{\partial f} & \frac{\partial f}{\partial \alpha} \frac{\partial \alpha}{\partial g} \\
\frac{\partial g}{\partial \alpha} \frac{\partial \alpha}{\partial f} & \frac{\partial g}{\partial \alpha} \frac{\partial \alpha}{\partial g}
\end{array}\right)=\left(\begin{array}{cc}
E_{M} & 0 \\
0 & E_{(N-M)}
\end{array}\right)
$$

by the chain rule of partial differentiations. Then we obtain

$$
\left(\frac{\partial f}{\partial \alpha}\right)\left(\frac{\partial \alpha}{\partial f}\right)=E_{M}
$$

for the rectangular matrices $\left(\frac{\partial f}{\partial \alpha}\right)$ and $\left(\frac{\partial \alpha}{\partial f}\right)$. 


\section{Appendix B: TDVP-EOM WITH COMPLEX PARAMETERS}

We consider complex TDVP-parameters $\left\{z_{i}, z_{i}^{*}\right\}_{i=1, n}[8]$. For the analytic wave function in Eq. (33), the TDVP Lagrangian[12] is defined as

$$
\begin{aligned}
L\left(z, \dot{z}, z^{*}, \dot{z}^{*}\right) & =\left\langle\Psi(z)|\hat{L}| \Psi(z)>=\left\langle\Psi(z)\left|\frac{i \hbar}{2}\left(\overrightarrow{\partial_{t}}-\overleftarrow{\partial_{t}}\right)-\hat{H}\right| \Psi(z)\right\rangle\right. \\
& =\frac{i \hbar}{2} \sum_{i=1}^{n}\left[\left\langle\Psi \mid \frac{\partial \Psi}{\partial z_{i}}\right\rangle \dot{z}_{i}-\dot{z}_{i}^{*}\left\langle\frac{\partial \Psi}{\partial z_{i}} \mid \Psi\right\rangle\right]+\langle\Psi|\hat{H}| \Psi\rangle
\end{aligned}
$$

We can obtain the TDVP-EOM by the least-action principle as

$$
\begin{aligned}
\delta J_{T D V P}= & \sum_{i=1}^{n} \int_{t_{1}}^{t_{2}} \delta z_{i}^{*}\left(i \hbar \sum_{j=1}^{n}\left\langle\frac{\partial \Psi}{\partial z_{i}} \mid \frac{\partial \Psi}{\partial z_{j}}\right\rangle \frac{d z_{j}}{d t}-\left\langle\frac{\partial \Psi}{\partial z_{i}}|\hat{H}| \Psi\right\rangle\right) d t \\
& +\sum_{i=1}^{n} \int_{t_{1}}^{t_{2}}\left(-i \hbar \sum_{j=1}^{n} \frac{d z_{j}^{*}}{d t}\left\langle\frac{\partial \Psi}{\partial z_{j}} \mid \frac{\partial \Psi}{\partial z_{i}}\right\rangle-\left\langle\Psi|\hat{H}| \frac{\partial \Psi}{\partial z_{i}}\right\rangle\right) \delta z_{i} d t=0,
\end{aligned}
$$

with fixed boundary conditions as $\delta z_{i}\left(t_{1}\right)=\delta z_{i}\left(t_{2}\right)=0$ and $\delta z_{i}^{*}\left(t_{1}\right)=\delta z_{i}^{*}\left(t_{2}\right)=0$. From Eq. (B2), we obtain the TDVP-EOM for the complex parameters $\left\{z_{i}(t), z_{i}^{*}(t)\right\}_{i=1, n}$ as

$$
i \hbar \sum_{j=1}^{n}\left\langle\frac{\partial \Psi}{\partial z_{i}} \mid \frac{\partial \Psi}{\partial z_{j}}\right\rangle \frac{d z_{j}}{d t}=\frac{\partial H}{\partial z_{i}^{*}}, \quad-i \hbar \sum_{j=1}^{n} \frac{d z_{j}^{*}}{d t}\left\langle\frac{\partial \Psi}{\partial z_{j}} \mid \frac{\partial \Psi}{\partial z_{i}}\right\rangle=\frac{\partial H}{\partial z_{i}}
$$

where $H=<\Psi|\hat{H}| \Psi>$.

If we consider analytic constraints as in Eq. (40) with Lagrange multipliers $\left\{\lambda_{a}\right\}_{a=1, m}$ and $\left\{\lambda_{a}^{*}\right\}_{a=1, m}$, we obtain the TDVP-EOM with the constraints as

$$
i \hbar \sum_{j=1}^{n}(\boldsymbol{C})_{i j} \dot{z}_{j}=\frac{\partial K}{\partial z_{i}^{*}}, \quad-i \hbar \sum_{j=1}^{n} \dot{z}_{j}^{*}(\boldsymbol{C})_{j i}=\frac{\partial K}{\partial z_{i}},
$$

where $(\boldsymbol{C})_{i j}=\left\langle\frac{\partial \Psi}{\partial z_{i}} \mid \frac{\partial \Psi}{\partial z_{j}}\right\rangle$ in Eq. (34) and the extended Hamiltonian is

$$
K\left(z, z^{*}\right)=H\left(z, z^{*}\right)+\sum_{a=1}^{m}\left[\lambda_{a} u_{a}(z)+\lambda_{a}^{*} u_{a}^{*}(z)\right] .
$$

The consistency condition for the constraints $u_{a}(z)=0$ in Eq. (40) is

$$
\dot{u}_{a}=\left\{u_{a}, H\right\}_{C G P B}+\sum_{b=1}^{m}\left\{u_{a}, u_{b}^{*}\right\}_{C G P B} \lambda_{b}^{*}=0 .
$$

With a column vector $\left(\boldsymbol{h}^{*}\right)_{a}=\left\{u_{a}, H\right\}_{C G P B}$, Eq. (B6) is rewritten in a matrix form as

$$
\boldsymbol{h}^{*}+\left\{\boldsymbol{u}, \boldsymbol{u}^{*}\right\}_{C G P B} \lambda^{*}=\mathbf{0}
$$


If the matrix $\left\{\boldsymbol{u}, \boldsymbol{u}^{*}\right\}_{C G P B}$ is nonsingular, we can obtain the Lagrange multipliers uniquely as

$$
\boldsymbol{\lambda}^{*}=-\left(\left\{\boldsymbol{u}, \boldsymbol{u}^{*}\right\}_{C G P B}\right)^{-1} \boldsymbol{h}^{*} .
$$

In the same way for the constraints $u_{a}^{*}(z)=0$ in Eq. (40), we have

$$
\boldsymbol{\lambda}=-\left(\left\{\boldsymbol{u}^{*}, \boldsymbol{u}\right\}_{C G P B}\right)^{-1} \boldsymbol{h} .
$$




\section{Appendix C: TRANSFORMATION OF COMPLEX PARAMETERS}

As in Appendix A, we consider the nonsingular transformation from the complex parameters $\left\{z_{i}\right\}_{i=1, n}$ to the parameters $\left\{u_{a}\right\}_{a=1, m}$ which will be frozen and their orthogonal complements $\left\{v_{b}\right\}_{b=1, n-m}$ as

$$
u_{a}=u_{a}\left(z_{1}, \ldots, z_{n}\right) \quad(a=1, \ldots, m<n), \quad v_{b}=v_{b}\left(z_{1}, \ldots, z_{n}\right) \quad(b=1, \ldots, n-m) .
$$

The transformation of variational freedoms is written in a matrix form

$$
\left(\begin{array}{l}
\delta u \\
\delta v
\end{array}\right)=\left(\begin{array}{l}
\frac{\partial u}{\partial z} \\
\frac{\partial v}{\partial z}
\end{array}\right) \delta z
$$

If the Jacobian matrix is nonsingular, we have

$$
E_{n}=\left(\begin{array}{l}
\frac{\partial u}{\partial z} \\
\frac{\partial v}{\partial z}
\end{array}\right)\left(\begin{array}{ll}
\frac{\partial z}{\partial u} & \frac{\partial z}{\partial v}
\end{array}\right)=\left(\begin{array}{ll}
\frac{\partial u}{\partial z} \frac{\partial z}{\partial u} & \frac{\partial u}{\partial z} \frac{\partial z}{\partial v} \\
\frac{\partial v}{\partial z} \frac{\partial z}{\partial u} & \frac{\partial v}{\partial z} \frac{\partial z}{\partial v}
\end{array}\right)=\left(\begin{array}{cc}
E_{m} & 0 \\
0 & E_{(n-m)}
\end{array}\right)
$$

by the chain rule of partial differentiations.

Moreover, if we can assume the orthogonality of local bases of the parameters $\left\{u_{a}\right\}_{a=1, m}$ and $\left\{v_{b}\right\}_{b=1, n-m}$ as

$$
\left\langle\frac{\partial \Psi}{\partial u} \mid \frac{\partial \Psi}{\partial v}\right\rangle=0, \quad\left\langle\frac{\partial \Psi}{\partial v} \mid \frac{\partial \Psi}{\partial u}\right\rangle=0,
$$

the Hermitian matrix $\boldsymbol{C}$ of Eq. (34) can be reduced as

$$
\begin{aligned}
& C=\left\langle\frac{\partial \Psi}{\partial z} \mid \frac{\partial \Psi}{\partial z}\right\rangle=\left(\left(\frac{\partial u}{\partial z}\right)^{\dagger}\left(\frac{\partial v}{\partial z}\right)^{\dagger}\right)\left(\begin{array}{l}
\left\langle\frac{\partial \Psi}{\partial u}\right. \\
\left\langle\frac{\partial \Psi}{\partial v}\right.
\end{array} \mid\right)\left(\left|\frac{\partial \Psi}{\partial u}\right\rangle\left|\frac{\partial \Psi}{\partial v}\right\rangle\right)\left(\begin{array}{l}
\frac{\partial u}{\partial z} \\
\frac{\partial v}{\partial z}
\end{array}\right) \\
& =\left(\begin{array}{ll}
\left(\frac{\partial u}{\partial z}\right)^{\dagger}\left(\frac{\partial v}{\partial z}\right)^{\dagger}
\end{array}\right)\left(\begin{array}{cc}
\left\langle\frac{\partial \Psi}{\partial u} \mid \frac{\partial \Psi}{\partial u}\right\rangle & 0 \\
0 & \left\langle\frac{\partial \Psi}{\partial v} \mid \frac{\partial \Psi}{\partial v}\right\rangle
\end{array}\right)\left(\begin{array}{l}
\frac{\partial u}{\partial z} \\
\frac{\partial v}{\partial z}
\end{array}\right) .
\end{aligned}
$$

From Eq. (C5), we can calculate the inverse as

$$
\begin{aligned}
C^{-1} & =\left(\begin{array}{ll}
\frac{\partial z}{\partial u} \frac{\partial z}{\partial v}
\end{array}\right)\left(\begin{array}{cc}
\left\langle\frac{\partial \Psi}{\partial u} \mid \frac{\partial \Psi}{\partial u}\right\rangle^{-1} & 0 \\
0 & \left\langle\frac{\partial \Psi}{\partial v} \mid \frac{\partial \Psi}{\partial v}\right\rangle^{-1}
\end{array}\right)\left(\begin{array}{c}
\left(\frac{\partial z}{\partial u}\right)^{\dagger} \\
\left(\frac{\partial z}{\partial v}\right)^{\dagger}
\end{array}\right) \\
& =\left(\frac{\partial z}{\partial u}\right)\left\langle\frac{\partial \Psi}{\partial u} \mid \frac{\partial \Psi}{\partial u}\right\rangle^{-1}\left(\frac{\partial z}{\partial u}\right)^{\dagger}+\left(\frac{\partial z}{\partial v}\right)\left\langle\frac{\partial \Psi}{\partial v} \mid \frac{\partial \Psi}{\partial v}\right\rangle^{-1}\left(\frac{\partial z}{\partial v}\right)^{\dagger}
\end{aligned}
$$




\section{Appendix D: INEQUALITY CONDITIONS FOR MOMENTS}

As shown in the example of $\S \mathrm{VB}$, real-valued constants $\mu_{r}$ for the moments of $r$-th power of a Hermitian operator $\hat{\omega}$ should satisfy the inequality[27]

$$
\left|\begin{array}{lllll}
\mu_{0} & \mu_{1} & \mu_{2} & \cdots & \mu_{k-1} \\
\mu_{1} & \mu_{2} & \mu_{3} & \cdots & \\
\mu_{2} & \mu_{3} & \mu_{4} & \cdots & \\
\cdots & \cdots & \cdots & \cdots & \cdots
\end{array}\right| \geq 0 \quad(k=1,2, \ldots)
$$

where $\mu_{0}=1$. The proof is as follows. For a normalized wave function $\Psi$, we consider a quadratic form whose coefficients are $M_{r s}=\mu_{r+s}$ as

$$
\begin{aligned}
\sum_{r, s=0}^{l} M_{r s} x_{r} x_{s} & =\sum_{r, s=0}^{l} \mu_{r+s} x_{r} x_{s}=\sum_{r, s=0}^{l}<\Psi\left|\hat{\omega}^{r+s}\right| \Psi>x_{r} x_{s} \\
& =\sum_{r, s=0}^{l}<\Psi\left|\hat{\omega}^{r} \hat{\omega}^{s}\right| \Psi>x_{r} x_{s}=\left\langle\Psi\left|\left[\sum_{r=0}^{l} \hat{\omega}^{r} x_{r}\right]^{2}\right| \Psi\right\rangle \geq 0 .
\end{aligned}
$$

As well known in linear algebra[26], for the non-negative quadratic form, all the principal minor determinants of coefficients $M_{r s}$ should be non-negative as

$$
\begin{aligned}
\left|\boldsymbol{M}_{k}\right| & =\left|\begin{array}{cccc}
M_{00} & M_{01} & \cdots & M_{0(k-1)} \\
M_{10} & M_{11} & \cdots & M_{1(k-1)} \\
\vdots & \vdots & & \vdots \\
M_{(k-1) 0} & M_{(k-1) 1} & \cdots & M_{(k-1)(k-1)}
\end{array}\right| \\
& =\left|\begin{array}{cccc}
\mu_{0} & \mu_{1} & \cdots & \mu_{k-1} \\
\mu_{1} & \mu_{2} & \cdots & \mu_{k} \\
\vdots & \vdots & & \vdots \\
\mu_{k-1} & \mu_{k} & \cdots & \mu_{2 k-2}
\end{array}\right| \geq 0 \quad(k=1, \ldots, l+1) .
\end{aligned}
$$

Since the inequality (D3) holds for any $l$, Eq. (D1) has been proven. 


\section{Appendix E: TRAJECTORIES BY POLAR COORDINATES}

We here use polar coordinates to examine the continuity of the trajectories at the origin in the numerical example of $\S \mathrm{VC} 1$. The polar coordinates are defined as $\theta=r \cos \gamma$ and $\eta=r \sin \gamma$. In Eq. (74), except for $\gamma=\frac{n \pi}{2}, n \pi+\frac{\pi}{4}$, the symplectic singularity does not occur at the origin as

$$
\lim _{r \rightarrow 0} \frac{1}{\sigma_{\theta \eta}}=\lim _{r \rightarrow 0}\left[\frac{1}{\hbar} \frac{\left(\theta^{2}+\eta^{2}\right)^{2}}{2 \theta \eta(\theta-\eta)}\right]=\lim _{r \rightarrow 0}\left[\frac{r}{2 \hbar \cos \gamma \sin \gamma(\cos \gamma-\sin \gamma)}\right]=0 .
$$

In the EOM's (75) and (76), we have also

$$
\begin{aligned}
\lim _{r \rightarrow 0} \dot{\theta}= & \lim _{r \rightarrow 0}\left(-\frac{1}{\hbar}\left[\frac{1}{\sin \gamma(\cos \gamma-\sin \gamma)}\right]\left\{\cos \gamma \sin \gamma\left(H_{11}-H_{22}\right)\right.\right. \\
& \left.\left.+\left[\left(\cos ^{2} \gamma-\sin ^{2} \gamma\right) \cos [r(\cos \gamma-\sin \gamma)]+r \sin \gamma \sin [r(\cos \gamma-\sin \gamma)]\right] H_{12}\right\}\right) \\
= & -\frac{1}{\hbar}\left[\frac{1}{\sin \gamma(\cos \gamma-\sin \gamma)}\right]\left[\cos \gamma \sin \gamma\left(H_{11}-H_{22}\right)+\left(\cos ^{2} \gamma-\sin ^{2} \gamma\right) H_{12}\right],
\end{aligned}
$$

and

$$
\begin{aligned}
\lim _{r \rightarrow 0} \dot{\eta}= & \lim _{r \rightarrow 0}\left(-\frac{1}{\hbar}\left[\frac{1}{\cos \gamma(\cos \gamma-\sin \gamma)}\right]\left\{\cos \gamma \sin \gamma\left(H_{11}-H_{22}\right)\right.\right. \\
& \left.\left.+\left[\left(\cos ^{2} \gamma-\sin ^{2} \gamma\right) \cos [r(\cos \gamma-\sin \gamma)]+r \cos \gamma \sin [r(\cos \gamma-\sin \gamma)]\right] H_{12}\right\}\right) \\
= & -\frac{1}{\hbar}\left[\frac{1}{\cos \gamma(\cos \gamma-\sin \gamma)}\right]\left[\cos \gamma \sin \gamma\left(H_{11}-H_{22}\right)+\left(\cos ^{2} \gamma-\sin ^{2} \gamma\right) H_{12}\right] .
\end{aligned}
$$

Then $\dot{\theta}$ and $\dot{\eta}$ do not diverge even when trajectories approach to the origin as $r \rightarrow 0$ except for $\gamma=\frac{n \pi}{2}, n \pi+\frac{\pi}{4}$. The trajectories can continuously go through the origin as shown in Figure 1. 
[1] E. Deumens, A. Diz, R. Longo, and Y. Öhrn, Rev. Mod. Phys. 66, 917 (1994), and references therein.

[2] P. A. M. Dirac, Proc. Cambridge Phil. Soc. 26, 376 (1930).

[3] J. Frenkel, Wave Mechanics, Advanced General Theory (Clarendon Press, Oxford, 1934).

[4] A. D. McLachlan, Mol. Phys. 8, 39 (1964).

[5] P. M. Morse, H. Feshbach, Methods of Theoretical Physics (McGraw-Hill, New York, 1953).

[6] A. K. Kerman and S. E. Koonin, Ann. Phys. 100, 332 (1976).

[7] D. J. Rowe, A. R. Ryman, and G. Rosensteel, Phys. Rev. A22, 2362 (1980).

[8] P. Kramer and M. Saraceno, Geometry of the Time-Dependent Variational Principle in Quantum Mechanics (Springer, New York, 1981).

[9] K. -K. Kan, Phys. Rev. A24, 2831 (1981).

[10] J. B. Broeckhove, L. Lathouwers, E. Kesteloot, and P. Van Leuven, Chem. Phys. Lett. 149, 547 (1988).

[11] K. Ohta, Chem. Phys. Lett. 329, 248 (2000).

[12] K. Ohta, Phys. Rev. A70, 022503 (2004).

[13] K. Ohta, Phys. Rev. A73, 044502 (2006).

[14] P. A. M. Dirac, Canad. J. Math., 2, 129 (1950).

[15] P. A. M. Dirac, Lectures on Quantum Mechanics (Yeshiva University Press, New York, 1964).

[16] A. Mukherji and M. Karplus, J. Chem. Phys. 38, 44 (1963).

[17] H. Ichikawa and H. Kagawa, Int. J. Quantum Chem. 52, 575 (1994).

[18] Q. Wu and T. V. Voorhis, Phys. Rev. A 72, 24502 (2005).

[19] T. Ogawa, M. Sumita, Y. Shimodo, and K. Morihashi, Chem. Phys. Lett. 511, 219 (2011).

[20] M. H. Henneaux and C. Teitelboim, Quantization of Gauge Systems (Princeton University Press, Princeton, New Jersey, 1992).

[21] O. Miskovic and J. Zanelli, J. Math. Phys. 44, 3876 (2003).

[22] J. Saavedra, R. Troncoso, and J. Zanelli, J. Math. Phys. 42, 4383 (2001).

[23] K. G. Kay, Chem. Phys. 137, 165 (1989).

[24] T. Fabcic, J. Main, and G. Wunner, J. Chem. Phys. 128, 044116 (2008).

[25] E. C. G. Sudarshan and N. Mukunda, Classical Dynamics: A Modern Perspective (John Wiley 
\& Sons, New York, 1974).

[26] G. Strang, Linear Algebra and its Applications (Academic Press, New York, 1976).

[27] K. Fushimi, Kakurituron Oyobi Toukeiron (Probability Theory and Statistics) (Kawade-syobou, Tokyo, 1942) [in Japanese]. 


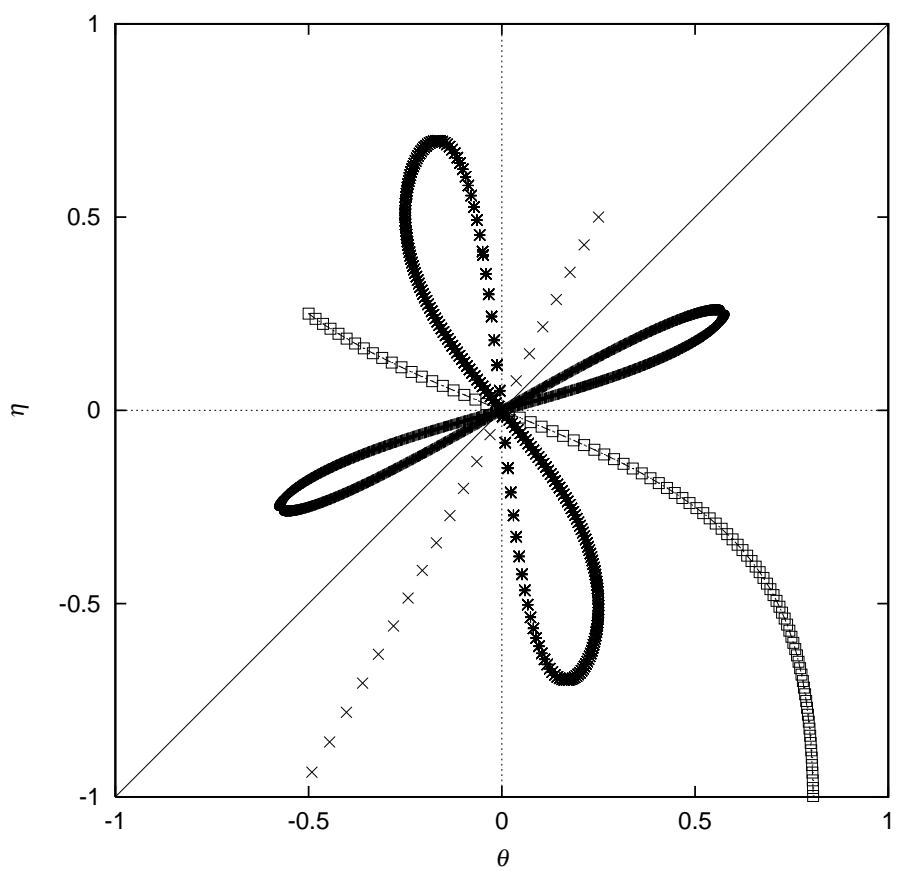

FIG. 1: Trajectories in $\theta-\eta$ space. Initial conditions are $\left(\theta_{0}, \eta_{0}\right)=( \pm 0.5, \pm 0.25)$, and $( \pm 0.25, \pm 0.5)$.

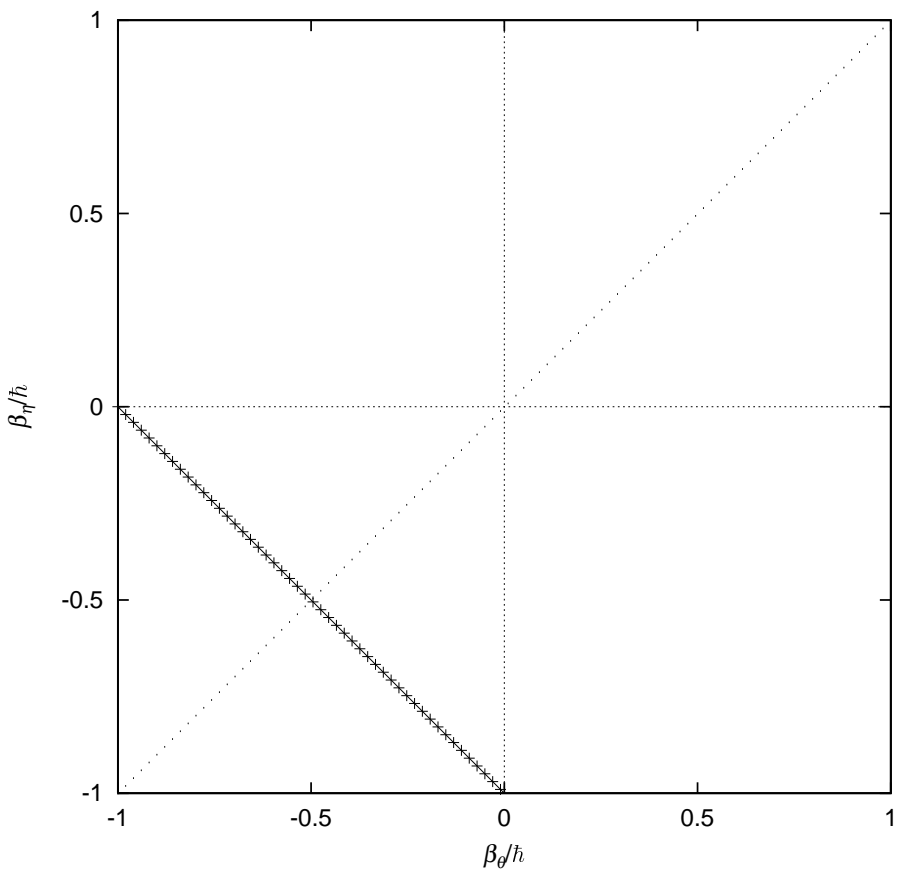

FIG. 2: Trajectories in $\beta_{\theta} / \hbar-\beta_{\eta} / \hbar$ space. 